\title{
On Reduction of Quaternionic Polynomials and Their Identical Equality
}

\author{
Dmytro A. Mierzejewski
}

\begin{abstract}
We show that any quaternionic polynomial with one variable can be represented in such a way that the number of its terms will be not larger than a certain number depending on the degree of the polynomial. We study also some particular cases where this number can be made even smaller. Then we use the above-mentioned representation to study how to check whether two given quaternionic polynomials with one variable are identically equal. We solve this problem for all linear polynomials and for some types of nonlinear polynomials.
\end{abstract}

Keywords. Quaternion, polynomial, quantic, equation.

\section{Introduction}

It is known that, due to non-commutativity of quaternions, the general form of a quaternionic polynomial (with one variable) is much more complicated that for a real or complex polynomial. Namely, even one term of a quaternionic polynomial may be of the form

$$
a^{(0)} x a^{(1)} x \ldots a^{(n-1)} x a^{(n)}
$$

without possibility to reduce (reduction is possible only if real coefficients are present); moreover a quaternionic polynomial may include many terms of the same degree, sometimes without possibility (and often without obvious possibility) to decrease their number.

The conception of polynomial, used in this paper, may look unusual for some part of the mathematical community, since they may define a polynomial as an algebraic object of the form

$$
a^{(n)} x^{n}+\cdots+a^{(1)} x+a^{(0)} .
$$

But dealing with non-commutative systems it is often better to understand a polynomial as it was explained above, while polynomials of the form (1) may be referred to with a certain adjective; the author of this paper usually 
calls them "classical polynomials", while other authors use words "simple", "unilateral", "regular".

The first main task of this paper is to describe a method allowing to decrease the number of the terms of a given polynomial. It turns out that every quaternionic polynomial of a given degree can be rewritten so that it may contain not more than a certain (depending on the degree) number of terms. This result is a simple generalization of [2] where only linear polynomials (more precisely, linear quantics) have been considered. We would like to mention also the work [13] described the same construction as in [2]. Just these two works inspired the author to fulfil investigations described in this paper.

Note also that decreasing the number of the terms of a polynomial is very useful when one looks for zeros of the polynomial. The point is that the process of looking for zeros of a quaternionic polynomial often demands very long calculations, and every additional term leads to a great additional piece of work. Therefore this paper may be considered as one on the topic about zeros of quaternionic polynomials and may be included in a list of works containing, for example, [11], [1], [12], [14], [3], [4], [10], [5], [15], [16], $[6],[7],[8],[9]$. Every work from this list includes investigations of some questions about zeros of quaternionic polynomials of some type(s). In some of those works polynomials with an arbitrary number of terms of a given degree are considered, but with no intention to decrease this number. It is quite possible that the decreasing can facilitate future investigations of zeros of quaternionic polynomials. But probably much more important and obviously very useful application of this reduction is when one looks for zeros of a concrete quaternionic polynomial with many terms.

The second main task of this paper is to describe a method to find out whether two given quaternionic polynomials are identically equal. For real or complex polynomials this problem is very simple. But for quaternionic polynomials this problem turns out to be much more difficult. It is possible to describe different transformations showing that the same quaternionic polynomials can be represented very differently. The simplest examples of such transformations are changes within terms provided by the fact that every real number commutes with every quaternion; for example, $4 j x(1+i) x k=j x(2+2 i) x 2 k$. Other examples are described just in this paper: the procedure of decreasing the number of the terms changes a polynomial very essentially.

But, on the other hand, just this procedure turns out to be useful for the second main task of this paper. Namely, we suppose that two given quaternionic polynomials are identically equal if and only if they coincide after being represented in a certain form described in this paper and called below "canonical". In this paper we have managed to prove this fact not for all quaternionic polynomials, but for several types of them, namely, for all linear ones and some part of nonlinear ones. 
Note also that the problem about identical equality of two polynomials is closely connected with the problem of whether a given polynomial is identically equal to zero, or, by other words, whether a given polynomial has its zeros in all points. Therefore the second main task of this paper may be considered as related to the problem of looking for zeros of quaternionic polynomials (as far as the first main task).

As for the structure of this paper, it may be divided into two parts, according to the two main tasks (though we do not reflect this fact formally). Section 7 is situated between these parts and in fact plays the role of preliminaries for the second part.

So, the first part is about the decreasing of the number of the terms. Firstly (in Section 4) we study a particular case of quaternionic polynomials and only later (in Section 5) pass to the general case. One may say that Section 4 is unnecessary since it describes a particular case of the construction of Section 5. But we have chosen such structure of the paper for two reasons. The first reason is our desire to make the text more understandable for the reader. The second reason is the fact that for the particular case described in Section 4 it is better to apply just the procedure of Section 4 rather than one of Section 5; this is important for practical application. Some further additional notes essential for application are contained by Section 6 . We pay much attention to application of the construction in the process of looking for zeros of a given quaternionic polynomial.

It is useful to note also that all the information from the first part of the paper can be easily generalized to a very wide class of hypercomplex systems instead of the system of quaternions. The point is that no multiplication of quaternionic imaginary units is involved in this part. But this paper is about only quaternionic polynomials, so that we do not write down explicitly such generalization.

The second part is about the checking whether polynomials are identically equal. In Section 8 we describe the corresponding theory for linear quaternionic polynomials, and in Section 9 we do the same for some types of nonlinear ones.

\section{Overview of Some Previous Works about Zeros of Quaternionic Polynomials}

[11] is a classical work in which roots of quaternions had been investigated in detail; in other words, the author had dealt with zeros of quaternionic polynomials of the form $x^{n}-a$. According to this work, the set of these zeros is either finite, or constitute a sphere.

In [1] it has been proved that for a certain class of quaternionic polynomials a proposition analogous to the fundamental theorem of algebra takes place, that is, every polynomial of this class has at least one zero. Namely, it is about polynomials having only one term of the greatest degree. 
During 2000s several works independently have appeared describing possible shapes of the sets of the zeros of any classical quaternionic polynomial (1). An example of such work is [12]. It turns out that the above-mentioned set is a union of finite numbers of isolated points and spheres with centers on the real axis (maybe, only points or only spheres, as particular (and so frequent) cases). In [3] the same facts have been discussed with different points of view; a useful detail of [3] is an explicit description of a method to find zeros of a given classical polynomial. In [10] some interesting additional investigations of classical quaternionic polynomials have been performed for the quadratic case; by the way, it was pointed out a simple class of polynomials connected with classical ones in a simple way and having a similar shape of the set of the zeros, but without some restrictions (for example, the centers of the spheres may be in any points, not only on the real axis).

Some information about zeros of several certain types of quaternionic polynomials (as a rule, not classical) has been obtained by a method of direct passing from a quaternionic equation to a system of four real equations (see the last paragraph of Section 3). The corresponding works are, for example, [14], [5], [15], [16], [6], [7], [8], [9]. These works contain much information about linear polynomials and some information about several kinds of quadratic ones. By the way, it is known from [14] that the set of the zeros of a linear quaternionic polynomial is always a linear manifold (if is not empty). As for quadratic quaternionic polynomials, sets of their zeros may consist of different interesting components being, for example, one- or two-dimensional quadrics.

\section{Preliminaries}

We use $\mathbb{H}$ as the standard notation for the set of all (real) quaternions. (We do not deal with so-called complex quaternions whose components are complex.) We use the notation $\mathbb{R}$ in its usual sense (the set of real numbers).

It is convenient for us to use two kinds of notations to denote quaternionic imaginary units. The first kind is the standard notations $i, j, k$; recall that $i^{2}=j^{2}=k^{2}=-1, \quad i j=-j i=k, j k=-k j=i, \quad k i=-i k=j$. But additionally we use notations $i_{1}, i_{2}, i_{3}$, where $i_{1}:=i, i_{2}:=j, i_{3}:=k$; moreover it is convenient to consider that $i_{0}:=1$.

We use subindices to denote the components of a quaternion as follows: $\mathbb{H} \ni \xi=\xi_{0}+\xi_{1} i+\xi_{2} j+\xi_{3} k$, where $\xi_{0}, \xi_{1}, \xi_{2}, \xi_{3} \in \mathbb{R}$. Therefore when an index at a letter is desirable for another aim we write it at the top and in round brackets (to do not mix with a power).

We want to clarify that we treat quaternionic polynomials as functions of quaternionic variable, and the definition of a quaternionic polynomial can be given, for example, by (10), where $x$ is the variable and all other letters denote given quaternions (see also the explanation situated at the beginning of Introduction). 
We always use the letter $x$ to denote the variable of a quaternionic polynomial. Since we consider only polynomials with one variable, we will omit the words "with one variable" writing about a quaternionic polynomial.

If a term of a quaternionic polynomial is of the form $a x^{\ell} b$ then we say that this term is non-split. If every non-zero term of a quaternionic polynomial is non-split then we say that it is a polynomial with non-split terms.

At last we should note about a simple procedure which is often used to investigate quaternionic polynomials. Any given quaternionic polynomial $f(x)$ can be rewritten as

$f_{0}\left(x_{0}, x_{1}, x_{2}, x_{3}\right)+f_{1}\left(x_{0}, x_{1}, x_{2}, x_{3}\right) i+f_{2}\left(x_{0}, x_{1}, x_{2}, x_{3}\right) j+f_{3}\left(x_{0}, x_{1}, x_{2}, x_{3}\right) k$,

where every $f_{t}$ is a real function of four real variables. To do this it is enough to rewrite all quaternions (including the variable $x$ ) by their components, to commit all necessary multiplications of quaternionic units, and to group all real numbers with respect to the quaternionic units. Note also that in this situation the equation $f(x)=0$ is equivalent to the following system of four real equations with four real unknowns:

$$
\left\{\begin{array}{l}
f_{0}\left(x_{0}, x_{1}, x_{2}, x_{3}\right)=0 \\
f_{1}\left(x_{0}, x_{1}, x_{2}, x_{3}\right)=0 \\
f_{2}\left(x_{0}, x_{1}, x_{2}, x_{3}\right)=0 \\
f_{3}\left(x_{0}, x_{1}, x_{2}, x_{3}\right)=0
\end{array}\right.
$$

When we use in this paper the procedure described in this paragraph then we will write about this very briefly calling this procedure rewriting by the components.

\section{The Reduction of Polynomials with Non-Split Terms}

In this section we will investigate quaternionic polynomials containing only non-split terms. The general form of such polynomial is

$$
\sum_{\mu=1}^{\nu^{(n)}} a^{(n, \mu)} x^{n} b^{(n, \mu)}+\cdots+\sum_{\mu=1}^{\nu^{(2)}} a^{(2, \mu)} x^{2} b^{(2, \mu)}+\sum_{\mu=1}^{\nu^{(1)}} a^{(1, \mu)} x b^{(1, \mu)}+c .
$$

Let us consider the fragment of the polynomial consisting of all terms of any given natural degree $m$ (that is, now we consider a quantic of the degree $m)$ :

$$
\sum_{\mu=1}^{\nu^{(m)}} a^{(m, \mu)} x^{m} b^{(m, \mu)} .
$$

Since in this section we will seldom write down the whole polynomial, it is convenient to simplify notations writing the fragment as follows:

$$
\sum_{\mu=1}^{\nu} a^{(\mu)} x^{m} b^{(\mu)}
$$


Now our aim is to represent (3) by as small as possible number of terms. It is easy to do quite analogously to [2] where the particular case of $m=1$ has been investigated. Namely:

$$
\begin{aligned}
& \sum_{\mu=1}^{\nu} a^{(\mu)} x^{m} b^{(\mu)}=\sum_{\mu=1}^{\nu} a^{(\mu)} x^{m}\left(b_{0}^{(\mu)}+b_{1}^{(\mu)} i+b_{2}^{(\mu)} j+b_{3}^{(\mu)} k\right) \\
= & \sum_{\mu=1}^{\nu}\left(a^{(\mu)} x^{m} b_{0}^{(\mu)}+a^{(\mu)} x^{m} b_{1}^{(\mu)} i+a^{(\mu)} x^{m} b_{2}^{(\mu)} j+a^{(\mu)} x^{m} b_{3}^{(\mu)} k\right) \\
= & \sum_{\mu=1}^{\nu}\left(a^{(\mu)} b_{0}^{(\mu)} x^{m}+a^{(\mu)} b_{1}^{(\mu)} x^{m} i+a^{(\mu)} b_{2}^{(\mu)} x^{m} j+a^{(\mu)} b_{3}^{(\mu)} x^{m} k\right) \\
= & \sum_{\mu=1}^{\nu} a^{(\mu)} b_{0}^{(\mu)} x^{m}+\sum_{\mu=1}^{\nu} a^{(\mu)} b_{1}^{(\mu)} x^{m} i+\sum_{\mu=1}^{\nu} a^{(\mu)} b_{2}^{(\mu)} x^{m} j+\sum_{\mu=1}^{\nu} a^{(\mu)} b_{3}^{(\mu)} x^{m} k \\
= & \left(\sum_{\mu=1}^{\nu} a^{(\mu)} b_{0}^{(\mu)}\right) x^{m}+\left(\sum_{\mu=1}^{\nu} a^{(\mu)} b_{1}^{(\mu)}\right) x^{m} i \\
& +\left(\sum_{\mu=1}^{\nu} a^{(\mu)} b_{2}^{(\mu)}\right) x^{m} j+\left(\sum_{\mu=1}^{\nu} a^{(\mu)} b_{3}^{(\mu)}\right) x^{m} k .
\end{aligned}
$$

Now all brackets contain only constants, so that the expression has only four terms and can be written as

$$
\alpha x^{m}+\beta x^{m} i+\gamma x^{m} j+\delta x^{m} k .
$$

Applying this method to every $m \in\{1, \ldots, n\}$ we get the whole polynomial (2) reduced to one with only $4 n+1$ terms:

$$
\sum_{m=1}^{n}\left(\alpha^{(m)} x^{m}+\beta^{(m)} x^{m} i+\gamma^{(m)} x^{m} j+\delta^{(m)} x^{m} k\right)+c,
$$

where for every $m \in\{1, \ldots, n\}$

$$
\begin{aligned}
\alpha^{(m)} & =\sum_{\mu=1}^{\nu} a^{(m, \mu)} b_{0}^{(m, \mu)}, & \beta^{(m)} & =\sum_{\mu=1}^{\nu} a^{(m, \mu)} b_{1}^{(m, \mu)}, \\
\gamma^{(m)} & =\sum_{\mu=1}^{\nu} a^{(m, \mu)} b_{2}^{(m, \mu)}, & \delta^{(m)} & =\sum_{\mu=1}^{\nu} a^{(m, \mu)} b_{3}^{(m, \mu)}
\end{aligned}
$$

Note that the formulas (6) are relatively simple in applying to reduction of certain given polynomials: one has only to add quaternionic coefficients multiplied by given real numbers.

It also should be mentioned that quite analogous reduction can be performed by decomposing the left coefficients $a^{(m, \mu)}$ (or $a^{(\mu)}$ ) instead of $b^{(m, \mu)} \quad\left(\right.$ or $\left.b^{(\mu)}\right)$. Then instead of (4) one gets

$$
x^{m} \varphi+i x^{m} \rho+j x^{m} \sigma+k x^{m} \tau,
$$

and instead of (5)

$$
\sum_{m=1}^{n}\left(x^{m} \varphi^{(m)}+i x^{m} \rho^{(m)}+j x^{m} \sigma^{(m)}+k x^{m} \tau^{(m)}\right)+c,
$$


where for every $m \in\{1, \ldots, n\}$

$$
\begin{aligned}
\varphi^{(m)} & =\sum_{\mu=1}^{\nu} a_{0}^{(m, \mu)} b^{(m, \mu)}, & & \rho^{(m)}=\sum_{\mu=1}^{\nu} a_{1}^{(m, \mu)} b^{(m, \mu)}, \\
\sigma^{(m)} & =\sum_{\mu=1}^{\nu} a_{2}^{(m, \mu)} b^{(m, \mu)}, & \tau^{(m)} & =\sum_{\mu=1}^{\nu} a_{3}^{(m, \mu)} b^{(m, \mu)} .
\end{aligned}
$$

Summarizing the above considerations we formulate the following theorem:

Theorem 1. Any quaternionic quantic of any natural degree, containing only non-split terms, can be written as a sum of not more than four terms. Therefore any quaternionic polynomial of any natural degree $n$, containing only non-split terms, can be written as a sum of not more than $4 n+1$ terms. Namely, (2) can be written as (5) using formulas (6) or as (8) using formulas (9).

\section{The Reduction in General Case}

It turns out that the T. Ell's method described in the previous section can be applied with only little changes to any quaternionic polynomials (that is, not only with non-split terms). Write down the general form of an arbitrary quaternionic polynomial:

$$
\begin{aligned}
& \sum_{\mu=1}^{\nu^{(n)}} a^{(n, 0, \mu)} x a^{(n, 1, \mu)} x \ldots x a^{(n, n-1, \mu)} x a^{(n, n, \mu)}+\ldots \\
& +\sum_{\mu=1}^{\nu^{(2)}} a^{(2,0, \mu)} x a^{(2,1, \mu)} x a^{(2,2, \mu)}+\sum_{\mu=1}^{\nu^{(1)}} a^{(1,0, \mu)} x a^{(1,1, \mu)}+c .
\end{aligned}
$$

Analogously to the previous section, we will consider the fragment of (10) consisting of all terms of any given degree $m$, and we will write this fragment with somewhat simplified notations:

$$
\sum_{\mu=1}^{\nu} a^{(0, \mu)} x a^{(1, \mu)} x \ldots x a^{(m-1, \mu)} x a^{(m, \mu)} .
$$

In the previous section two ways to simplify the fragment were described (depending on whether left or right coefficients are decomposed). Now the number of the ways will be $m+1$, according to the number of coefficients in each term. The point is that in each term we will decompose every coefficient excepting one; the difference between the ways is determined by the position of these not decomposed coefficients. Now we will describe all ways simultaneously considering that the not decomposed coefficients are $a^{(\ell, \mu)}$ with a fixed $\ell \in\{0, \ldots, m\}$ and all $\mu \in\{1, \ldots, \nu\}$ (we stress that $\ell$ is fixed, that is, we deal with the same position in every term). 
Firstly note that decomposing the coefficients $a^{(m, \mu)}$ one obtains the following (similarly to the previous section):

$$
\begin{aligned}
& \sum_{\mu=1}^{\nu} a^{(0, \mu)} x a^{(1, \mu)} x \ldots x a^{(m-1, \mu)} x a^{(m, \mu)} \\
& =\left(\sum_{\mu=1}^{\nu} a^{(0, \mu)} x a^{(1, \mu)} x \ldots x a^{(m-1, \mu)} a_{0}^{(m, \mu)}\right) x \\
& \quad+\left(\sum_{\mu=1}^{\nu} a^{(0, \mu)} x a^{(1, \mu)} x \ldots x a^{(m-1, \mu)} a_{1}^{(m, \mu)}\right) x i \\
& \quad+\left(\sum_{\mu=1}^{\nu} a^{(0, \mu)} x a^{(1, \mu)} x \ldots x a^{(m-1, \mu)} a_{2}^{(m, \mu)}\right) x j \\
& \quad+\left(\sum_{\mu=1}^{\nu} a^{(0, \mu)} x a^{(1, \mu)} x \ldots x a^{(m-1, \mu)} a_{3}^{(m, \mu)}\right) x k .
\end{aligned}
$$

So, we have obtained four addends (as in the previous section), but now the sums in the brackets are not constants.

Nevertheless all these sums are of the form quite similar to (11), but with the degree $m-1$ instead of $m$. Thus it is possible to rewrite every sum decomposing again the last coefficients of their terms. Note that it is possible to consider that these last coefficients are $a^{(m-1, \mu)}$ since the second multiplier in every case is a real number and can be moved even to the beginning of the expression. After performing all this with every sum we get 16 addends with the following form of each addend:

$$
\left(\sum_{\mu=1}^{\nu} a^{(0, \mu)} x a^{(1, \mu)} x \ldots x a^{(m-2, \mu)} a_{r}^{(m-1, \mu)} a_{p}^{(m, \mu)}\right) x i_{r} x i_{p}
$$

here we have used the alternative notations for the basis of $\mathbb{H}$ (see Section 3).

It is understandable that one can continue this construction further quite analogously. Moreover it is possible to begin analogous procedure from the left moving to the right. Of course, it is also possible to cover several steps from the left and then several steps from the right. And therefore with any given $\ell \in\{0, \ldots, m\}$ it is possible to decompose step by step all coefficients excepting $a^{(\ell, \mu)}$ and to get as a result $4^{m}$ terms of the form

$$
i_{p^{(0)}} x \ldots i_{p^{(\ell-1)}} x\left(\sum_{\mu=1}^{\nu} a_{p^{(0)}}^{(0, \mu)} \ldots a_{p^{(\ell-1)}}^{(\ell-1, \mu)} a^{(\ell, \mu)} a_{p^{(\ell+1)}}^{(\ell+1, \mu)} \ldots a_{p^{(m)}}^{(m, \mu)}\right) x i_{p^{(\ell+1)}} \ldots x i_{p^{(m)}} .
$$

Here the sum is already a constant, so that (12) represents one term and the considered fragment is reduced to one containing just $4^{m}$ terms, which can be written as follows:

$$
\sum_{p^{(0)}, \ldots, p^{(\ell-1)}, p^{(\ell+1)}, \ldots, p^{(m)}=0}^{3} i_{p^{(0)}} x \ldots i_{p^{(\ell-1)}} x \alpha^{\left(p^{(0)}, \ldots, p^{(\ell-1)}, p^{(\ell+1)}, \ldots, p^{(m)}\right)} x i_{p^{(\ell+1)}} \ldots x i_{p^{(m)}},
$$


where for every $p^{(0)}, \ldots, p^{(\ell-1)}, p^{(\ell+1)}, \ldots, p^{(m)} \in\{0,1,2,3\}$

$$
\begin{aligned}
& \alpha^{\left(p^{(0)}, \ldots, p^{(\ell-1)}, p^{(\ell+1)}, \ldots, p^{(m)}\right)} \\
& =\sum_{\mu=1}^{\nu} a_{p^{(0)}}^{(0, \mu)} a_{p^{(1)}}^{(1, \mu)} \ldots a_{p^{(\ell-1)}}^{(\ell-1, \mu)} a^{(\ell, \mu)} a_{p^{(\ell+1)}}^{(\ell+1, \mu)} \ldots a_{p^{(m)}}^{(m, \mu)} .
\end{aligned}
$$

Note that though the formulas (13) and (14) look somewhat complicated, the corresponding calculations are again comparatively simple since to calculate $\alpha^{\left(p^{(0)}, \ldots, p^{(\ell-1)}, p^{(\ell+1)}, \ldots, p^{(m)}\right)}$ one has only to multiply quaternions by real numbers and to add quaternions; no multiplication of two imaginary units is involved.

Recall that we have dealt with a quantic being a fragment of the given polynomial. Applying this method to the whole polynomial of the degree $n$ (by considering all corresponding fragments) one can get a polynomial with the following number of terms:

$$
1+4+4^{2}+\cdots+4^{n}=\frac{4^{n+1}-1}{3} .
$$

Summarizing the above considerations we formulate the following theorem:

Theorem 2. Any quaternionic quantic of any natural degree $m$ can be written as a sum of not more than $4^{m}$ terms. Namely, choosing any $\ell \in\{0, \ldots, m\}$, one can rewrite (11) as (13) using formula (14). This procedure allows to rewrite any quaternionic polynomial of any natural degree $n$ as a sum of not more than $\frac{4^{n+1}-1}{3}$ terms.

Remark 1. If all coefficients in some position are written without a certain quaternionic unit (for example, are of the form $a_{0}+a_{2} j+a_{3} k$ ) then this position generates decomposition into less than 4 terms and there is less than $4^{m}$ terms in the result. Namely, given respectively $q^{(0)}, q^{(1)}, \ldots$, $q^{(\ell-1)}, q^{(\ell)}, q^{(\ell+1)}, \ldots, q^{(m)}$ quaternionic units in the positions we get $q^{(0)} q^{(1)} \ldots q^{(\ell-1)} q^{(\ell+1)} \ldots q^{(m)}$ terms in the result (for the quantic).

\section{Practical Items on the Reduction}

As it was already mentioned in Introduction, the procedure described in Sections 4 and 5 may be very useful when one has to solve a certain polynomial quaternionic equation. For a great majority of types of such equations we do not know any method of solution better than rewriting by the components, although sometimes with some additional manipulations (see [14], [5], [15], [16], [6], [7], [8], [9]). This paper proposes one more type of additional manipulations making it possible to decrease the number of the terms in a given equation. Now we will consider more carefully how one should perform these manipulations before looking for zeros of a given quaternionic polynomial.

Note first of all that, according to Sections 4 and 5, we deal in fact with a quantic rather than with an arbitrary polynomial. So, firstly one has to break 
up the given polynomial into fragments each from which contains terms of the same degree. Every such fragment has to be considered separately.

Moreover we consider that the fragment of a natural degree is already given in a form which cannot be simplified by "very elementary" methods. Namely, it should be represented just in the form (11) and moreover: 1) any coefficient equal to 0 or 1 should not be written; 2) any real number in any term should be moved to be multiplied by another coefficient of the term if such coefficient exists; it should be moved to the beginning of the term if it is alone there; 3 ) if $a^{(1)}, \ldots, a^{(s)}$ are any quaternions and $\Pi, \Pi^{(1)}, \Pi^{(2)}$ are any products of any number of quaternions and copies of the unknown $x$ then instead of $a^{(1)} \Pi+\cdots+a^{(s)} \Pi$ there should be $\left(a^{(1)}+\cdots+a^{(s)}\right) \Pi$, instead of $\Pi a^{(1)}+\cdots+\Pi a^{(s)}$ there should be $\Pi\left(a^{(1)}+\cdots+a^{(s)}\right)$, instead of $\Pi^{(1)} a^{(1)} \Pi^{(2)}+\cdots+\Pi^{(1)} a^{(s)} \Pi^{(2)}$ there should be $\Pi^{(1)}\left(a^{(1)}+\cdots+a^{(s)}\right) \Pi^{(2)}$.

The next question is about structure of the terms of the fragment. If all their terms are non-split then the proper procedure is one of Sections 4 . Otherwise the procedure of Sections 5 may be proper, but in fact in some cases some additional simplification is possible. For example, if every term is of the form $a x^{2} b x c$ then it is unnecessary to treat them as ax1xbxc, but it is better to perform the procedure the same way as for axbxc changing $x$ by $x^{2}$ in all corresponding positions. Analogously one can deal with a fragment consisting of terms of the form

$$
a^{(0)} x^{\lambda^{(1)}} a^{(1)} x^{\lambda^{(2)}} \ldots a^{(u-1)} x^{\lambda^{(u)}} a^{(u)}
$$

(with fixed $\lambda^{(1)}, \ldots, \lambda^{(u)}$ ) the same way as with one containing any terms of the degree $u$; it is easy to understand how the corresponding procedure looks, but we do not describe it explicitly to avoid too complicated notations. But note that this simplified procedure is in fact the same as the standard procedure of Section 5 taking into account Remark 1: one can write the coefficient 1 in all corresponding positions and "decompose" it into one addend in every position obtaining in the end just $4^{u}$ terms. Moreover in the case $a^{(0)}=1$ or $a^{(u)}=1$ (in every term of the fragment) one has again theoretically the same situation, but practically it is better at once to move out of brackets respectively $x^{\lambda^{(1)}}$ or $x^{\lambda^{(u)}}$.

After analysing the structure of the terms the next step is to choose the position of coefficients which will not be decomposed and simultaneously to decide whether the procedure is really useful (since sometimes it is possible that the initial fragment would be simpler than the final one). It is possible to propose some numerical characterizations for degree of difficulty and to construct some formulas allowing to choose the best way. But in this paper we will not propose any such characterization keeping in mind that different methods of calculation may need different characterizations; by the way, calculation by a computer may essentially differ from manual one, and there may be essential difference between computer programs used to perform the considered tasks. Besides we would like to tell a hypothesis that sometimes it would be useful to break up a fragment into shorter fragments and to deal 
with them separately; of course, in order to study this question one has to choose some certain rules for the estimations that is not done in this paper.

So, after making all above mentioned steps one should simplify the fragment (or decide to do not change it). One should do all this with every fragment of the given polynomial before performing further procedures to look for its zeros.

\section{On the Terminology of the Below Sections}

So, the information from the previous sections implies that every quaternionic polynomial can be represented, in particular, in the following form:

$$
\begin{aligned}
& \sum_{\tau^{(1)}, \ldots, \tau^{(n)}=0}^{3} a^{\left(n, \tau^{(1)}, \tau^{(2)}, \ldots, \tau^{(n)}\right)} x i_{\tau^{(1)}} x i_{\tau^{(2)}} \ldots x i_{\tau^{(n)}}+\ldots \\
& +\sum_{\tau^{(1)}, \tau^{(2)}=0}^{3} a^{\left(2, \tau^{(1)}, \tau^{(2)}\right)} x i_{\tau^{(1)}} x i_{\tau^{(2)}}+\sum_{\tau=0}^{3} a^{(1, \tau)} x i_{\tau}+a^{(0)} .
\end{aligned}
$$

Note that now we have chosen one variant of those described above, namely, such that all coefficients which may be arbitrary quaternions are written on the left-hand side. We will call this representation the canonical form of the polynomial.

Considering a quaternionic polynomial given in the canonical form we will use somewhat non-standard meaning of the word "coefficient". Namely, only quaternions denoted in (16) by the letter $a$ with indices will be called coefficients of the polynomial. By other words, coefficients are those constants from the canonical form which may be arbitrary; while the quaternionic units $i_{\tau}$ occupying their stable positions are not called coefficients.

If two quaternionic polynomials in the canonical form are given then considering a coefficient of the first polynomial it is possible to refer to the corresponding coefficient of the second one; it means the coefficient situated at the same combination of copies of $x$ and quaternionic units.

Below we will use somewhat non-standard meaning of the concept of the degree of a polynomial. Namely, we will name this way the largest degree of a term of the polynomial, even if the coefficient of this term equals zero. Therefore the same polynomial can be written differently providing different degrees. This meaning is inconvenient in many situations, but it turns out to be convenient in this paper, simplifying some descriptions. The words "linear" and "quadratic" will mean respectively "of the degree 1" and "of the degree 2" just in our sense of the concept "degree".

It should be noted also that when we refer to every coefficient of a quaternionic polynomial of a given degree in the canonical form then we will mean coefficients at all possible for this degree combinations of copies of $x$ and quaternionic units. If some combination is absent then the coefficient at it equals zero. This remark is necessary to be precise in some propositions concerning comparison of two polynomials. 


\section{The Identical Equality of Linear Polynomials}

Theorem 3. A linear quaternionic polynomial represented in the canonical form

$$
a x+b x i+c x j+d x k+h
$$

is identically equal to zero if and only if $a=b=c=d=h=0$.

Proof. Here the only non-trivial implication is the following: if the polynomial is identically equal to zero then $a=b=c=d=h=0$. So, suppose that

$$
\forall x \in \mathbb{H} \quad a x+b x i+c x j+d x k+h=0 .
$$

First of all, putting $x=0$ we easily get $h=0$.

Therefore it is enough to consider the quantic

$$
a x+b x i+c x j+d x k .
$$

Rewriting by the components we get the following form of this polynomial:

$$
\begin{aligned}
& \left(\left(a_{0}-b_{1}-c_{2}-d_{3}\right) x_{0}+\left(-a_{1}-b_{0}-c_{3}+d_{2}\right) x_{1}\right. \\
& \left.+\left(-a_{2}+b_{3}-c_{0}-d_{1}\right) x_{2}+\left(-a_{3}-b_{2}+c_{1}-d_{0}\right) x_{3}\right) \\
& +\left(\left(a_{1}+b_{0}-c_{3}+d_{2}\right) x_{0}+\left(a_{0}-b_{1}+c_{2}+d_{3}\right) x_{1}\right. \\
& \left.+\left(-a_{3}-b_{2}-c_{1}+d_{0}\right) x_{2}+\left(a_{2}-b_{3}-c_{0}-d_{1}\right) x_{3}\right) i \\
& +\left(\left(a_{2}+b_{3}+c_{0}-d_{1}\right) x_{0}+\left(a_{3}-b_{2}-c_{1}-d_{0}\right) x_{1}\right. \\
& \left.+\left(a_{0}+b_{1}-c_{2}+d_{3}\right) x_{2}+\left(-a_{1}+b_{0}-c_{3}-d_{2}\right) x_{3}\right) j \\
& +\left(\left(a_{3}-b_{2}+c_{1}+d_{0}\right) x_{0}+\left(-a_{2}-b_{3}+c_{0}-d_{1}\right) x_{1}\right. \\
& \left.+\left(a_{1}-b_{0}-c_{3}-d_{2}\right) x_{2}+\left(a_{0}+b_{1}+c_{2}-d_{3}\right) x_{3}\right) k
\end{aligned}
$$

Then we can write the following system of four equalities being true for every real $x_{0}, x_{1}, x_{2}, x_{3}$ :

$$
\left\{\begin{array}{c}
\left(a_{0}-b_{1}-c_{2}-d_{3}\right) x_{0}+\left(-a_{1}-b_{0}-c_{3}+d_{2}\right) x_{1} \\
\quad+\left(-a_{2}+b_{3}-c_{0}-d_{1}\right) x_{2}+\left(-a_{3}-b_{2}+c_{1}-d_{0}\right) x_{3}=0, \\
\left(a_{1}+b_{0}-c_{3}+d_{2}\right) x_{0}+\left(a_{0}-b_{1}+c_{2}+d_{3}\right) x_{1} \\
\quad+\left(-a_{3}-b_{2}-c_{1}+d_{0}\right) x_{2}+\left(a_{2}-b_{3}-c_{0}-d_{1}\right) x_{3}=0, \\
\left(a_{2}+b_{3}+c_{0}-d_{1}\right) x_{0}+\left(a_{3}-b_{2}-c_{1}-d_{0}\right) x_{1} \\
\quad+\left(a_{0}+b_{1}-c_{2}+d_{3}\right) x_{2}+\left(-a_{1}+b_{0}-c_{3}-d_{2}\right) x_{3}=0, \\
\left(a_{3}-b_{2}+c_{1}+d_{0}\right) x_{0}+\left(-a_{2}-b_{3}+c_{0}-d_{1}\right) x_{1} \\
\quad+\left(a_{1}-b_{0}-c_{3}-d_{2}\right) x_{2}+\left(a_{0}+b_{1}+c_{2}-d_{3}\right) x_{3}=0 .
\end{array}\right.
$$

Treating (17) as a system of linear equations with respect to $x_{0}, x_{1}, x_{2}$, $x_{3}$ one easily comes to the conclusion that every coefficient of this system equals 0 .

By other words, every pair of brackets in (17) contains an expression equal to 0 . If one writes down all corresponding equalities treating all letters with indices as unknowns then one gets a system of 16 linear equations with 16 unknowns; the square matrix of this system is the following (if the order 
of the unknowns is $a_{0}, a_{1}, a_{2}, a_{3}, b_{0}, b_{1}, b_{2}, b_{3}, c_{0}, c_{1}, c_{2}, c_{3}, d_{0}$, $\left.d_{1}, d_{2}, d_{3}\right)$ :

$$
\left(\begin{array}{cccccccccccccccc}
1 & 0 & 0 & 0 & 0 & -1 & 0 & 0 & 0 & 0 & -1 & 0 & 0 & 0 & 0 & -1 \\
0 & -1 & 0 & 0 & -1 & 0 & 0 & 0 & 0 & 0 & 0 & -1 & 0 & 0 & 1 & 0 \\
0 & 0 & -1 & 0 & 0 & 0 & 0 & 1 & -1 & 0 & 0 & 0 & 0 & -1 & 0 & 0 \\
0 & 0 & 0 & -1 & 0 & 0 & -1 & 0 & 0 & 1 & 0 & 0 & -1 & 0 & 0 & 0 \\
0 & 1 & 0 & 0 & 1 & 0 & 0 & 0 & 0 & 0 & 0 & -1 & 0 & 0 & 1 & 0 \\
1 & 0 & 0 & 0 & 0 & -1 & 0 & 0 & 0 & 0 & 1 & 0 & 0 & 0 & 0 & 1 \\
0 & 0 & 0 & -1 & 0 & 0 & -1 & 0 & 0 & -1 & 0 & 0 & 1 & 0 & 0 & 0 \\
0 & 0 & 1 & 0 & 0 & 0 & 0 & -1 & -1 & 0 & 0 & 0 & 0 & -1 & 0 & 0 \\
0 & 0 & 1 & 0 & 0 & 0 & 0 & 1 & 1 & 0 & 0 & 0 & 0 & -1 & 0 & 0 \\
0 & 0 & 0 & 1 & 0 & 0 & -1 & 0 & 0 & -1 & 0 & 0 & -1 & 0 & 0 & 0 \\
1 & 0 & 0 & 0 & 0 & 1 & 0 & 0 & 0 & 0 & -1 & 0 & 0 & 0 & 0 & 1 \\
0 & -1 & 0 & 0 & 1 & 0 & 0 & 0 & 0 & 0 & 0 & -1 & 0 & 0 & -1 & 0 \\
0 & 0 & 0 & 1 & 0 & 0 & -1 & 0 & 0 & 1 & 0 & 0 & 1 & 0 & 0 & 0 \\
0 & 0 & -1 & 0 & 0 & 0 & 0 & -1 & 1 & 0 & 0 & 0 & 0 & -1 & 0 & 0 \\
0 & 1 & 0 & 0 & -1 & 0 & 0 & 0 & 0 & 0 & 0 & -1 & 0 & 0 & -1 & 0 \\
1 & 0 & 0 & 0 & 0 & 1 & 0 & 0 & 0 & 0 & 1 & 0 & 0 & 0 & 0 & -1
\end{array}\right)
$$

The determinant of this matrix equals $2^{16}=65536$ (this fact is quite checkable even by manual direct calculation). Since it does not equal 0, the system of 16 linear equations has only one solution, and this solution is

$$
\begin{aligned}
& a_{0}=a_{1}=a_{2}=a_{3}=b_{0}=b_{1}=b_{2}=b_{3}=c_{0}=c_{1}=c_{2}=c_{3} \\
& =d_{0}=d_{1}=d_{2}=d_{3}=0,
\end{aligned}
$$

but it means that $a=b=c=d=0$, and the theorem is proved.

Corollary 1. Let two linear quaternionic polynomials written in the canonical form be given: $a x+b x i+c x j+d x k+h$ and $\alpha x+\beta x i+\gamma x j+\delta x k+\lambda$. Then

$$
\forall x \in \mathbb{H} \quad a x+b x i+c x j+d x k+h=\alpha x+\beta x i+\gamma x j+\delta x k+\lambda
$$

if and only if

$$
a=\alpha, \quad b=\beta, \quad c=\gamma, \quad d=\delta, \quad h=\lambda .
$$

For the proof it is enough to observe that subtracting the polynomials one obtains the identical zero, but simultaneously the difference equals

$$
(a-\alpha) x+(b-\beta) x i+(c-\gamma) x j+(d-\delta) x k+(h-\lambda) .
$$

\section{The Identical Equality of Nonlinear Polynomials of Some Types}

Lemma 1. Let the following quaternionic polynomial be given:

$$
a x^{2}+b x^{2} i+c x^{2} j+d x^{2} k .
$$

If this polynomial is identically equal to zero, then

$$
a=b=c=d=0 .
$$


Proof. Thinking analogously to the proof of Theorem 3 one gets a system of equalities being true for every $x_{0}, x_{1}, x_{2}, x_{3} \in \mathbb{R}$. Taking into account that $x^{2}=\left(x_{0}^{2}-x_{1}^{2}-x_{2}^{2}-x_{3}^{2}\right)+2 x_{0} x_{1} i+2 x_{0} x_{2} j+2 x_{0} x_{3} k$, it is easy to write down this system replacing in (17) $x_{0}$ with $x_{0}^{2}-x_{1}^{2}-x_{2}^{2}-x_{3}^{2}, x_{1}$ with $2 x_{0} x_{1}$, $x_{2}$ with $2 x_{0} x_{2}$, and $x_{3}$ with $2 x_{0} x_{3}$ :

$$
\left\{\begin{array}{c}
\left(a_{0}-b_{1}-c_{2}-d_{3}\right)\left(x_{0}^{2}-x_{1}^{2}-x_{2}^{2}-x_{3}^{2}\right)+\left(-a_{1}-b_{0}-c_{3}+d_{2}\right) 2 x_{0} x_{1} \\
\quad+\left(-a_{2}+b_{3}-c_{0}-d_{1}\right) 2 x_{0} x_{2}+\left(-a_{3}-b_{2}+c_{1}-d_{0}\right) 2 x_{0} x_{3}=0, \\
\left(a_{1}+b_{0}-c_{3}+d_{2}\right)\left(x_{0}^{2}-x_{1}^{2}-x_{2}^{2}-x_{3}^{2}\right)+\left(a_{0}-b_{1}+c_{2}+d_{3}\right) 2 x_{0} x_{1} \\
\quad+\left(-a_{3}-b_{2}-c_{1}+d_{0}\right) 2 x_{0} x_{2}+\left(a_{2}-b_{3}-c_{0}-d_{1}\right) 2 x_{0} x_{3}=0, \\
\left(a_{2}+b_{3}+c_{0}-d_{1}\right)\left(x_{0}^{2}-x_{1}^{2}-x_{2}^{2}-x_{3}^{2}\right)+\left(a_{3}-b_{2}-c_{1}-d_{0}\right) 2 x_{0} x_{1} \\
\quad+\left(a_{0}+b_{1}-c_{2}+d_{3}\right) 2 x_{0} x_{2}+\left(-a_{1}+b_{0}-c_{3}-d_{2}\right) 2 x_{0} x_{3}=0, \\
\left(a_{3}-b_{2}+c_{1}+d_{0}\right)\left(x_{0}^{2}-x_{1}^{2}-x_{2}^{2}-x_{3}^{2}\right)+\left(-a_{2}-b_{3}+c_{0}-d_{1}\right) 2 x_{0} x_{1} \\
\quad+\left(a_{1}-b_{0}-c_{3}-d_{2}\right) 2 x_{0} x_{2}+\left(a_{0}+b_{1}+c_{2}-d_{3}\right) 2 x_{0} x_{3}=0 .
\end{array}\right.
$$

Putting $x_{0}^{2}-x_{1}^{2}-x_{2}^{2}-x_{3}^{2}=: y_{0}, 2 x_{0} x_{1}=: y_{1}, 2 x_{0} x_{2}=: y_{2}$, and $2 x_{0} x_{3}=: y_{3}$ we can rewrite this system in the following form:

$$
\left\{\begin{array}{c}
\left(a_{0}-b_{1}-c_{2}-d_{3}\right) y_{0}+\left(-a_{1}-b_{0}-c_{3}+d_{2}\right) y_{1} \\
\quad+\left(-a_{2}+b_{3}-c_{0}-d_{1}\right) y_{2}+\left(-a_{3}-b_{2}+c_{1}-d_{0}\right) y_{3}=0 \\
\left(a_{1}+b_{0}-c_{3}+d_{2}\right) y_{0}+\left(a_{0}-b_{1}+c_{2}+d_{3}\right) y_{1} \\
\quad+\left(-a_{3}-b_{2}-c_{1}+d_{0}\right) y_{2}+\left(a_{2}-b_{3}-c_{0}-d_{1}\right) y_{3}=0 \\
\left(a_{2}+b_{3}+c_{0}-d_{1}\right) y_{0}+\left(a_{3}-b_{2}-c_{1}-d_{0}\right) y_{1} \\
\quad+\left(a_{0}+b_{1}-c_{2}+d_{3}\right) y_{2}+\left(-a_{1}+b_{0}-c_{3}-d_{2}\right) y_{3}=0 \\
\left(a_{3}-b_{2}+c_{1}+d_{0}\right) y_{0}+\left(-a_{2}-b_{3}+c_{0}-d_{1}\right) y_{1} \\
\quad+\left(a_{1}-b_{0}-c_{3}-d_{2}\right) y_{2}+\left(a_{0}+b_{1}+c_{2}-d_{3}\right) y_{3}=0 .
\end{array}\right.
$$

We know that these four equalities are true for every $x_{0}, x_{1}, x_{2}, x_{3} \in \mathbb{R}$. Let us establish what it means in terms of $y_{0}, y_{1}, y_{2}, y_{3} \in \mathbb{R}$. For this aim write down the corresponding relations as a system:

$$
\left\{\begin{array}{l}
x_{0}^{2}-x_{1}^{2}-x_{2}^{2}-x_{3}^{2}=y_{0}, \\
2 x_{0} x_{1}=y_{1} \\
2 x_{0} x_{2}=y_{2} \\
2 x_{0} x_{3}=y_{3} .
\end{array}\right.
$$

Let us check whether it is possible to find $x_{0}, x_{1}, x_{2}, x_{3} \in \mathbb{R}$ being suitable for given $y_{0}, y_{1}, y_{2}, y_{3} \in \mathbb{R}$. Let us restrict this consideration taking $y_{0}>0$. So, let $y_{0}, y_{1}, y_{2}, y_{3} \in \mathbb{R}$ be given, where $y_{0}>0$. Then from (19) we get:

$$
x_{1}=\frac{y_{1}}{2 x_{0}}, \quad x_{2}=\frac{y_{2}}{2 x_{0}}, \quad x_{3}=\frac{y_{3}}{2 x_{0}},
$$

if $x_{0} \neq 0$. Then

$$
x_{0}^{2}-\frac{y_{1}^{2}}{4 x_{0}^{2}}-\frac{y_{2}^{2}}{4 x_{0}^{2}}-\frac{y_{3}^{2}}{4 x_{0}^{2}}=y_{0} .
$$

From the last equation one can calculate a suitable value of $x_{0}$ rewriting the equation as biquadratic one:

$$
4 x_{0}^{4}-4 y_{0} x_{0}^{2}-\left(y_{1}^{2}+y_{2}^{2}+y_{3}^{2}\right)=0,
$$


the discriminant

$$
\begin{gathered}
D=16\left(1+y_{1}^{2}+y_{2}^{2}+y_{3}^{2}\right)>0, \\
x_{0}^{2}=\frac{y_{0}+\sqrt{1+y_{1}^{2}+y_{2}^{2}+y_{3}^{2}}}{2}>0, \\
x_{0}=\sqrt{\frac{y_{0}+\sqrt{1+y_{1}^{2}+y_{2}^{2}+y_{3}^{2}}}{2}}>0
\end{gathered}
$$

(recall that we need at least one suitable $x_{0}$, so that it is unnecessary to write down all solutions of the biquadratic equation). Since here the abovementioned condition $x_{0} \neq 0$ holds true, on the next step one can calculate $x_{1}, x_{2}, x_{3}$ by (20).

Now, knowing that (18) is true for every $x_{0}, x_{1}, x_{2}, x_{3} \in \mathbb{R}$, we see that it is true for every $y_{0}>0$ and for every $y_{1}, y_{2}, y_{3} \in \mathbb{R}$. But (18) is a linear system with respect to $y_{0}, y_{1}, y_{2}, y_{3}$, so that the set of its solutions is a linear manifold. The only linear manifold containing all points $\left(y_{0}, y_{1}, y_{2}, y_{3}\right)$ with $y_{0}>0$ and $y_{1}, y_{2}, y_{3} \in \mathbb{R}$ is the entire four-dimensional space. Therefore all coefficients of (18) have to equal zero since only in this case the entire four-dimensional space is the set of the solutions. So, we have obtained just the same situation as in the proof of Theorem 3, and the same considerations as in that proof lead us to the conclusion that $a=b=c=d=0$.

Theorem 4. Let a quadratic quaternionic polynomial with non-split terms, written in the canonical form, be given:

$$
a x^{2}+b x^{2} i+c x^{2} j+d x^{2} k+p x+q x i+r x j+s x k+h .
$$

Then this polynomial is identically equal to zero if and only if

$$
a=b=c=d=p=q=r=s=h=0 .
$$

Proof. Again it is enough to prove that the identical equality to zero implies the zero coefficients. Thus we suppose that the polynomial is identically equal to zero.

Substituting $2 x$ instead of $x$ we can write:

$$
\forall x \in \mathbb{H} \quad 4 a x^{2}+4 b x^{2} i+4 c x^{2} j+4 d x^{2} k+2 p x+2 q x i+2 r x j+2 s x k+h=0 .
$$

Besides multiplying the given polynomial by 4 we can write:

$\forall x \in \mathbb{H} \quad 4 a x^{2}+4 b x^{2} i+4 c x^{2} j+4 d x^{2} k+4 p x+4 q x i+4 r x j+4 s x k+4 h=0$.

Subtract (21) from (22):

$$
\forall x \in \mathbb{H} \quad 2 p x+2 q x i+2 r x j+2 s x k+3 h=0 .
$$

Since the last polynomial is linear and represented in the canonical form, it is possible to apply Theorem 3 and to write: $p=q=r=s=h=0$.

So, the given polynomial can be written as $a x^{2}+b x^{2} i+c x^{2} j+d x^{2} k$, but then it is possible to apply Lemma 1 and to write: $a=b=c=d=0$. 
Corollary 2. Two quadratic quaternionic polynomials with non-split terms, written in the canonical form, are identically equal if and only if every coefficient of the first polynomial equals the corresponding coefficient of the second polynomial.

The proof is quite similar to the one of Corollary 1.

Remark 2. According to Theorem 1, the canonical form of any quaternionic polynomial with non-split terms is again with non-split terms. Therefore Theorem 4 or Corollary 2 can be used to investigate any quadratic quaternionic polynomials with non-split terms: rewriting them in the canonical form one gets polynomials suitable for Theorem 4 or Corollary 2.

Theorem 5. Let a quaternionic polynomial of a natural degree $n$ written in the canonical form be given. Suppose that for every $p \in\{2, \ldots, n\}$ the polynomial contains not more than one non-zero term of the degree $p$ :

$$
\begin{aligned}
& a^{(n)} x i_{t^{(n, 1)}} \ldots x i_{t^{(n, n)}}+a^{(n-1)} x i_{t^{(n-1,1)}} \ldots x i_{t^{(n-1, n-1)}} \\
& +\cdots+a^{(2)} x i_{t^{(2,1)}} x i_{t^{(2,2)}}+\sum_{\tau=0}^{3} a^{(1, \tau)} x i_{\tau}+a^{(0)} .
\end{aligned}
$$

Then this polynomial is identically equal to zero if and only if every its coefficient equals zero.

Proof. We will prove this theorem by induction with respect to the degree $n$. Theorem 3 implies that for $n=1$ the theorem is true. Then we suppose that it is true for $n=m-1$ and will deduce that it is true for $n=m$.

Analogously to previous proofs, it is enough to prove that the identical equality to zero implies the zero coefficients. Thus we suppose that the given polynomial with $n=m$ is identically equal to zero.

Substituting $2 x$ instead of $x$ we can write:

$$
\begin{aligned}
& \forall x \in \mathbb{H} \\
& 2^{m} a^{(m)} x i_{t^{(m, 1)}} \ldots x i_{t^{(m, m)}}+2^{m-1} a^{(m-1)} x i_{t^{(m-1,1)}} \ldots x i_{t^{(m-1, m-1)}} \\
& +\cdots+4 a^{(2)} x i_{t^{(2,1)}} x i_{t^{(2,2)}}+2 \sum_{\tau=0}^{3} a^{(1, \tau)} x i_{\tau}+a^{(0)}=0 .
\end{aligned}
$$

Multiplying the given polynomial by $2^{m}$ we can write:

$$
\begin{aligned}
& \forall x \in \mathbb{H} \\
& 2^{m} a^{(m)} x i_{t^{(m, 1)}} \ldots x i_{t^{(m, m)}}+2^{m} a^{(m-1)} x i_{t^{(m-1,1)}} \ldots x i_{t^{(m-1, m-1)}} \\
& +\cdots+2^{m} a^{(2)} x i_{t^{(2,1)}} x i_{t^{(2,2)}}+2^{m} \sum_{\tau=0}^{3} a^{(1, \tau)} x i_{\tau}+2^{m} a^{(0)}=0 .
\end{aligned}
$$

Subtracting (23) from (24) we get:

$$
\begin{aligned}
& \forall x \in \mathbb{H} \\
& \left(2^{m}-2^{m-1}\right) a^{(m-1)} x i_{t^{(m-1,1)}} \ldots x i_{t^{(m-1, m-1)}}+\ldots \\
& +\left(2^{m}-4\right) a^{(2)} x i_{t^{(2,1)}} x i_{t^{(2,2)}}+\left(2^{m}-2\right) \sum_{\tau=0}^{3} a^{(1, \tau)} x i_{\tau} \\
& +\left(2^{m}-1\right) a^{(0)}=0 .
\end{aligned}
$$


The polynomial in (25) is of the degree $m-1$ and satisfies the condition of the theorem. Therefore, according to the assumption, every coefficient of (25) equals zero. This fact implies that the considered polynomial of the degree $m$ can be written as the following monomial: $a^{(m)} x i_{t^{(m, 1)}} \ldots x i_{t^{(m, m)}}$. It is clear that this monomial can be identically equal to zero only if $a^{(m)}=0$, so that we have already proved that all coefficients equal zero.

Corollary 3. Let two quaternionic polynomials of a natural degree $n$ written in the canonical form be given. And let each of them have the following property: for every $p \in\{2, \ldots, n\}$ the polynomial contains not more than one non-zero term of the degree p. Moreover suppose that both polynomials have the same combinations of quaternionic units in their non-zero terms. Then these polynomials are identically equal if and only if every coefficient of the first polynomial equals the corresponding coefficient of the second one.

The proof is again quite similar to the proofs of Corollaries 1 and 2 .

Theorem 6. Let a quaternionic polynomial of a natural degree $n$ written in the canonical form be given. Suppose that for every $p \in\{3, \ldots, n\}$ the polynomial contains not more than one non-zero term of the degree $p$ and besides every quadratic term is non-split, that is, the polynomial is of the following form:

$$
\begin{aligned}
& a^{(n)} x i_{t^{(n, 1)}} \ldots x i_{t^{(n, n)}}+a^{(n-1)} x i_{t^{(n-1,1)}} \ldots x i_{t^{(n-1, n-1)}}+\ldots \\
& +a^{(3)} x i_{t^{(3,1)}} x i_{t^{(3,2)}} x i_{t^{(3,3)}}+\sum_{\tau=0}^{3} a^{(2, \tau)} x^{2} i_{\tau}+\sum_{\tau=0}^{3} a^{(1, \tau)} x i_{\tau}+a^{(0)} .
\end{aligned}
$$

Then this polynomial is identically equal to zero if and only if every its coefficient equals zero.

The proof is analogous to the one of Theorem 5, using Theorem 4 instead of Theorem 3.

Corollary 4. Let two quaternionic polynomials be given, and let each of them be of the form (26). Moreover suppose that both polynomials have the same combinations of quaternionic units in their non-zero terms. Then these polynomials are identically equal if and only if every coefficient of the first polynomial equals the corresponding coefficient of the second one.

\section{Some Final Remarks}

So, the author sees two kinds of mathematical activity for which information from this paper may be very useful. The first one is looking for zeros of concrete long quaternionic polynomials, especially if it is performed by rewriting by the components; then decreasing the number of the terms of the polynomial may be very fruitful method to simplify calculations. The second one is finding out whether two given quaternionic polynomials are identically equal. 
In both cases further investigations on the topic would be very desirable. Namely, for looking for zeros of polynomials it is useful to work out an algorithm to choose the best way of transformations, as it was discussed in Section 6.

As for finding out whether polynomials are identically equal, it is desirable to widen the class of polynomials for which the method is proved to be correct. Besides it is desirable to investigate this method for the cases of other positions of the not decomposed coefficients (while only the case of the left position is investigated in this paper); maybe, it would be useful to have a choice and to choose the best way, analogously to transformations before looking for zeros.

\section{Acknowledgements}

The author is thankful to Dr O. F. Gerus and V. S. Szpakowski for useful discussions.

\section{References}

[1] S. Eilenberg, I. Niven, The "fundamental theorem of algebra" for quaternions. Bulletin of the American Mathematical Society. Vol. 50 no. 4 1944, pp. 246-248.

[2] T. Ell, On systems of linear quaternionic functions. Preprint, February 2007. URL http://www.arxiv.org/abs/math/0702084v1

[3] G. Gentili, C. Stoppato, Zeros of regular functions and polynomials of a quaternionic variable. Michigan Mathematical Journal. Vol. 56 2008, pp. 655-667.

[4] D. Janovská, G. Opfer, Linear equations in quaternionic variables. Mitteilungen der Mathematischen Gesselschaft in Hamburg. Vol. 27 2008, pp. 223-234.

[5] D. Mierzejewski, Investigation of quaternionic quadratic equations I. Factorization and passing to a system of real equations. Bulletin de la Société des Sciences et des Lettres de Łódź. Série: Recherches sur les Déformations. Vol. 56 2008, pp. 17-26.

[6] D. Mierzejewski, Quasi-spherical and multi-quasi-spherical polynomial quaternionic equations: introduction of the notions and some examples. Adv. appl. Clifford alg. Online DOI: 10.1007/s00006-010-0250-8.

[7] D. Mierzejewski, Linear manifolds in sets of solutions of quaternionic polynomial equations of several types. Adv. appl. Clifford alg. Online DOI: 10.1007/s00006-010-0252-6.

[8] D. Mierzejewski, Spheres in sets of solutions of quadratic quaternionic equations of some types. Bulletin de la Société des Sciences et des Lettres de Łódź. Série: Recherches sur les Déformations. To appear.

[9] D. Mierzejewski, The dimensions of sections of the sets of the solutions of some quadratic quaternionic equations. Bulletin de la Société des Sciences et des Lettres de Łódź. Série: Recherches sur les Déformations. To appear.

[10] D. Mierzejewski, V. Szpakowski, On solutions of some types of quaternionic quadratic equations. Bulletin de la Société des Sciences et des Lettres de Łódź. Série: Recherches sur les Déformations. Vol. 55 2008, pp. 49-58. 
[11] I. Niven, The roots of a quaternion. American Mathematical Monthly. Vol. 49, 1942, pp. 386-388.

[12] A. Pogorui, M. Shapiro, On the structure of the set of the zeros of quaternionic polynomials. Complex Variables and Elliptic Equations. Vol. 49 no. 6 2004, pp. 379-389.

[13] S. Sangwine, Canonic form of linear quaternion functions. Preprint, January 2008. URL http://arxiv.org/abs/0801.2887

[14] V. Szpakowski, Solution of general linear quaternionic equations. The XI Kravchuk International Scientific Conference. Kyiv (Kiev), Ukraine, 2006, p. 661. (In Ukrainian.)

[15] V. Szpakowski, Solution of general quadratic quaternionic equations. Bulletin de la Société des Sciences et des Lettres de Łódź. Série: Recherches sur les Déformations. Vol. 58 2009, pp. 45-58.

[16] V. Shpakivskyi (Szpakowski), Linear quaternionic equations and their systems. Adv. in appl. Clifford alg. Online DOI: 10.1007/s00006-010-0264-2.

Dmytro A. Mierzejewski

Department of Mathematics

Kielce University of Technology

Tysiạclecia Państwa Polskiego Avenue 7, building C

25-314 Kielce

Poland

e-mail: dmytro1972@gmail.com

Received: June 12, 2010.

Revised: December 30, 2010.

Accepted: March 14, 2011.

Open Access This article is distributed under the terms of the Creative Commons Attribution Noncommercial License which permits any noncommercial use, distribution, and reproduction in any medium, provided the original author(s) and source are credited. 